\title{
QUANTUM $j$-INVARIANT IN POSITIVE CHARACTERISTIC II: FORMULAS AND VALUES AT THE QUADRATICS
}

\author{
L. DEMANGOS AND T.M. GENDRON
}

\begin{abstract}
In this sequel to [2, the multi-valued quantum $j$-invariant in positive characteristic is studied at quadratic elements. For every quadratic $f$, an explicit expression for each of the values of $j^{\mathrm{qt}}(f)$ is given as a limit of rational functions of $f$. It is proved that the number of values of $j^{\mathrm{qt}}(f)$ is finite.
\end{abstract}

\section{INTRODUCTION}

Let $\mathbb{F}_{q}$ be the field with $q$ elements, $q=p^{r}$ a prime power, $k=\mathbb{F}_{q}(T)$ the function field over $\mathbb{F}_{q}, k_{\infty}$ the completion of $k$ with respect to the valuation $v(f)=$ $-\operatorname{deg}_{T}(f)$. In [2], the quantum $j$-invariant was introduced as a multi-valued modular function

$$
j^{\mathrm{qt}}: \mathrm{GL}_{2}(A) \backslash k_{\infty} \multimap k_{\infty} \cup\{\infty\}
$$

obtained as the limit of a sequence of approximating functions

$$
j_{\varepsilon}: k_{\infty} \longrightarrow k_{\infty} \cup\{\infty\}
$$

as $\varepsilon \rightarrow 0$. Explicit formulas for the approximants (10) in terms of the sequence $\left\{\mathrm{q}_{i}\right\} \subset A$ of best approximations of $f$ were obtained and using them, it was shown that $f \in k$ if and only if eventually $j_{\varepsilon}(f)=\infty$.

In this paper we consider $j^{\text {qt }}(f)$ in the special case of $f \in k_{\infty}$ quadratic. Here we are able to derive explicit formulas not just for the approximants but for all the values of $j^{\mathrm{qt}}(f)$, each of which expressed as a limit of certain rational functions of $f$. Using these formulas we are able to prove that for $f$ quadratic, $\# j^{\text {at }}(f)<\infty$.

In what follows, we fix notation used in 2 .

\section{Values at Quadratic Units}

Fix $a \in A-\mathbb{F}_{q}, b \in \mathbb{F}_{q}^{\times}$with

$$
d:=\operatorname{deg} a>0
$$

and write $D=a^{2}+4 b$. The solutions $f, f^{\prime}$ of $X^{2}-a X-b=0$ are quadratic units, and every quadratic unit in $k_{\infty}-k$ occurs in this way. Since $f f^{\prime}=-b$, we have $\left|f f^{\prime}\right|=1$. Moreover, since $f+f^{\prime}=a$, we have as well that $|f|,\left|f^{\prime}\right| \neq 1$. Therefore, if we assume that $|f|>1$, then

$$
|f|=|a|=q^{d}>1 \quad \text { and } \quad\left|f^{\prime}\right|=|b / a|=q^{-d}<1 .
$$

Consider the recursive sequence

$$
\mathrm{Q}_{0}=1, \mathbf{Q}_{1}=a, \ldots, \mathbf{Q}_{n+1}=a \mathbf{Q}_{n}+b \mathbf{Q}_{n-1} .
$$

1991 Mathematics Subject Classification. Primary 11R58,11F03,11R11; Secondary 11K60.

Key words and phrases. quantum j-invariant, global function fields, quadratic extensions, Diophantine approximation. 
Note that when $b=1, \mathrm{Q}_{n}=\mathrm{q}_{n}$ is the sequence of best approximations for $f=$ $[a, a, \ldots]$; see $\S 3$ of [2]. It is clear that for all $n$

$$
\left|\mathbf{Q}_{n}\right|=|a|^{n}=q^{d n} \text {. }
$$

Recall Binet's formula

$$
\mathbf{Q}_{n}=\frac{f^{n+1}-\left(f^{\prime}\right)^{n+1}}{\sqrt{D}}, \quad n=0,1, \ldots
$$

which, while usually stated over $\mathbb{Q}$, is equally true in this setting, by a very simple induction on $n$. The root $\sqrt{D}$ is chosen so that in odd characteristic, $f=(a+$ $\sqrt{D}) / 2$; in even characteristic, we simply take $\sqrt{D}=a$.

Recall that $\|x\|=$ distance of $x$ to the nearest element of $A$; see [2], $\S 2$ for this and other relevant notation. Binet's formula immediately gives

$$
\left\|\mathrm{Q}_{n} f\right\|=q^{-(n+1) d}:
$$

indeed,

$$
\left|Q_{n} f-Q_{n+1}\right|=\frac{\left|f^{\prime}\right|^{n+1}\left|f-f^{\prime}\right|}{|\sqrt{D}|}=\left|f^{\prime}\right|^{n+1}=q^{-(n+1) d}<1 .
$$

In particular, $\mathrm{Q}_{n}^{\perp}=\mathrm{Q}_{n+1}$, where $\lambda^{\perp}$ denotes the element of $A$ closest to $\lambda f$.

The remainder of this section is devoted to deriving an explicit formula for each value of $j^{\mathrm{qt}}(f)$. In view of our need to work with monic polynomials, we make the following modification to the above sequence. For each $n$, we write $\overline{\mathbf{Q}}_{n}$ for the unique monic polynomial obtained as $c_{n} \mathbf{Q}_{n}$ for some $c_{n} \in \mathbb{F}_{q}^{\times}$. Then $\left|\bar{Q}_{n}\right|=\left|\mathbf{Q}_{n}\right|$ and $\left\|\overline{\mathbf{Q}}_{n} f\right\|=\left\|\mathrm{Q}_{n} f\right\|$ since $\overline{\mathbf{Q}}_{n}^{\perp}=c_{n} \mathbf{Q}_{n}^{\perp}$. (N.B. $\overline{\mathbf{Q}}_{n}^{\perp}$ is not necessarily monic.) Note that if we choose $c \in \mathbb{F}_{q}^{\times}$so that $c a$ is monic then $c_{n}=c^{n}$. It follows from Binet's formula that

$$
\overline{\mathbf{Q}}_{n}=c^{n} \frac{f^{n+1}-\left(f^{\prime}\right)^{n+1}}{\sqrt{D}}=\frac{\bar{f}^{n+1}-\left(\bar{f}^{\prime}\right)^{n+1}}{c \sqrt{D}}, \quad \bar{f}:=c f, \quad \bar{f}^{\prime}:=c f^{\prime} .
$$

The set

$$
\mathcal{B}=\left\{T^{d-1} \overline{\mathrm{Q}}_{0}, \ldots, T \overline{\mathrm{Q}}_{0}, \overline{\mathrm{Q}}_{0} ; T^{d-1} \overline{\mathrm{Q}}_{1}, \ldots, T \overline{\mathrm{Q}}_{1}, \overline{\mathrm{Q}}_{1} ; \ldots\right\}
$$

is a basis for $A$ as an $\mathbb{F}_{q}$-vector space: as the degree map deg : $\mathcal{B} \rightarrow \mathbb{N}=\{0,1, \ldots\}$ is a bijection. The order in which we have presented the elements of $\mathcal{B}$ corresponds to decreasing errors: for $0 \leq l \leq d-1$ and $n \geq 0$

$$
\left\|T^{l} \overline{\mathrm{Q}}_{n} f\right\|=\left|T^{l} \overline{\mathrm{Q}}_{n} f-T^{l} \overline{\mathrm{Q}}_{n+1}\right|=q^{l-(n+1) d}<1 .
$$

In particular, $\left(T^{l} \overline{\mathbf{Q}}_{n}\right)^{\perp}=T^{l} \overline{\mathbf{Q}}_{n}^{\perp}$, and the map

$$
\mathcal{B} \longrightarrow q^{-\mathbb{N}}, \quad T^{l} \overline{\mathrm{Q}}_{n} \longmapsto\left\|T^{l} \overline{\mathrm{Q}}_{n} f\right\|
$$

defines a bijection between $\mathcal{B}$ and the set of possible errors.

Write

$$
\mathcal{B}(i)=\left\{T^{d-1} \overline{\mathbf{Q}}_{i}, \ldots, \overline{\mathbf{Q}}_{i}\right\}
$$

for the $i$ th block of $\mathcal{B}$. Furthermore, for $0 \leq \tilde{d} \leq d-1$, denote

$$
\mathcal{B}(i)_{\tilde{d}}=\left\{T^{\tilde{d}} \overline{\mathbf{Q}}_{i}, \ldots, \overline{\mathbf{Q}}_{i}\right\} .
$$


Lemma 1. Let $l \in\{0, \ldots, d-1\}$ and write

$$
d_{l}=d-1-l .
$$

Then

$$
\Lambda_{q^{-N d-l}}(f)=\operatorname{span}_{\mathbb{F}_{q}}\left(\mathcal{B}(N)_{d_{l}}, \mathcal{B}(N+1), \ldots\right) .
$$

Proof. First observe that

$$
\operatorname{span}_{\mathbb{F}_{q}}\left(\mathcal{B}(N)_{d_{l}}, \mathcal{B}(N+1), \ldots\right) \subset \Lambda_{q^{-N d-l}}(f) .
$$

Indeed, for $T^{\tilde{d}} \overline{\mathbf{Q}}_{N+r}$ with $\tilde{d} \leq d-1$, and $\tilde{d} \leq d_{l}$ if $r=0$, we have

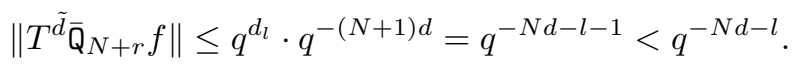

Moreover, by (6),$\Lambda_{q^{-N d-l}}(f)$ contains no other elements of $\mathcal{B}$. In view of the bijection (7), no linear combination of the excluded basis elements could appear in $\Lambda_{q^{-N d-l}}(f)$. This proves the equality in the statement of the Lemma.

We recall the main definitions established in [2]. For each $\varepsilon, \Lambda_{\varepsilon}^{\operatorname{mon}}(f)$ is the subset of monic polynomials in $\Lambda_{\varepsilon}(f)$. We defined

$$
\zeta_{f, \varepsilon}(n):=\sum_{\lambda \in \Lambda_{\varepsilon}^{\operatorname{mon}}(f)-\{0\}} \lambda^{-n}, \quad n \in \mathbb{N},
$$

and

$$
j_{\varepsilon}(f)=\frac{1}{\frac{1}{T^{q}-T}-J_{\varepsilon}(f)},
$$

where

$$
J_{\varepsilon}(f)=\frac{T^{q^{2}}-T}{\left(T^{q}-T\right)^{q+1}} \cdot \tilde{J}_{\varepsilon}(f), \quad \tilde{J}_{\varepsilon}(f):=\frac{\zeta_{f, \varepsilon}\left(q^{2}-1\right)}{\zeta_{f, \varepsilon}(q-1)^{q+1}} .
$$

Then $j^{\text {qt }}(f)$ is the set of limits of $j_{\varepsilon}(f)$ for $\varepsilon \rightarrow 0$. It follows from Lemma 1 that to calculate the values of $j^{\text {qt }}(f)$, it suffices to consider the possible limits of $j_{\varepsilon}(f)$ formed from the spans of the initial truncations of $\mathcal{B}$.

Let $\varepsilon=q^{-N d-l}$. As in [2], we decompose

$$
\Lambda_{\varepsilon}^{\text {mon }}(f)=\Lambda_{\varepsilon}^{\text {bas }}(f) \bigsqcup \Lambda_{\varepsilon}^{\text {non-bas }}(f)
$$

where

$$
\Lambda_{\varepsilon}^{\text {bas }}(f)=\left\{\mathcal{B}(N)_{d_{l}}, \mathcal{B}(N+1), \ldots\right\}
$$

and

$$
\Lambda_{\varepsilon}^{\text {non-bas }}(f)=\Lambda_{\varepsilon}^{\text {mon }}(f)-\left(\Lambda_{\varepsilon}^{\text {bas }}(f) \cup\{0\}\right) .
$$

Any element $\lambda \in \Lambda_{\varepsilon}^{\text {non-bas }}(f)$ may therefore be written in the form

$$
\lambda=c_{0}(T) \overline{\mathbf{Q}}_{N}+c_{1}(T) \overline{\mathbf{Q}}_{N+1}+\cdots+c_{m}(T) \overline{\mathbf{Q}}_{N+m},
$$

where the $c_{i}(T) \in A$ are polynomials that are not all zero and satisfy the following conditions

I. $c_{m}(T)$ is monic and if $\lambda=c_{m}(T) \overline{\mathrm{Q}}_{N+m}, c_{m}(T) \neq T^{j}$ for all $j \leq d-1$.

II. $\operatorname{deg}\left(c_{i}(T)\right) \leq d-1, i=0, \ldots, m$.

III. $\operatorname{deg}\left(c_{0}(T)\right) \leq d_{l}$. 
In view of this characterization, sums over $\lambda \in \Lambda_{\varepsilon}^{\text {non-bas }}(f)$ may be understood as sums indexed by tuples $c_{0}(T), \ldots, c_{m}(T)$ subject to conditions I, II and III, and so we will abbreviate

$$
\sum_{\substack{\text { Conditions } \\ \text { I,IIIIII }}}:=\sum_{\substack{\text { non-bas } \\ \Lambda_{\varepsilon}^{\text {non }}(f)}}
$$

Using this notation, we may now express

$$
\tilde{J}_{\mathcal{E}}(f)=\frac{\sum_{\lambda \in \Lambda_{\varepsilon}^{\text {bas }}(f)} \lambda^{1-q^{2}}+\sum_{\substack{\text { conditions } \\ \mathrm{I}, \mathrm{II}, \mathrm{III}}}\left(\sum_{i=0}^{m} c_{i}(T) \overline{\mathrm{Q}}_{N+i}\right)^{1-q^{2}}}{\left(\sum_{\lambda \in \Lambda_{\varepsilon}^{\text {bas }}(f)} \lambda^{1-q}+\sum_{\substack{\text { conditions } \\ \mathrm{I}, \mathrm{II}, \mathrm{III}}}\left(\sum_{i=0}^{m} c_{i}(T) \overline{\mathrm{Q}}_{N+i}\right)^{1-q}\right)^{q+1}} .
$$

Write

$$
\zeta_{T, l}(n):=1+T^{-n}+\cdots+T^{-n d_{l}}, \quad \zeta_{T}(n):=\zeta_{T, 0}(n),
$$

then define

$$
H_{l}(n):=\zeta_{T, l}(n)+\sum_{\substack{\text { Conditions I,II,III } \\ c_{0}(T) \neq 0}}\left(\sum_{i=0}^{m} c_{i}(T) \bar{f}^{i}\right)^{-n}
$$

and

$$
H(n):=\frac{\zeta_{T}(n)}{\bar{f}^{n}-1}+\sum_{\substack{\text { Conditions } \\ \mathrm{I}, \mathrm{II}}}\left(\sum_{i=1}^{m} c_{i}(T) \bar{f}^{i}\right)^{-n}
$$

Now let

$$
\tilde{J}(f)_{l}:=\frac{H_{l}\left(q^{2}-1\right)+H\left(q^{2}-1\right)}{\left(H_{l}(q-1)+H(q-1)\right)^{1+q}} .
$$

Note that $\tilde{J}(f)_{l}$ converges: indeed both numerator and denominator have absolute value 1 , and $\left|\tilde{J}(f)_{l}\right|=1$. Finally, we write

$$
J(f)_{l}:=\frac{T^{q^{2}}-T}{\left(T^{q}-T\right)^{q+1}} \cdot \tilde{J}(f)_{l} .
$$

Theorem 1. Let $f$ be a quadratic unit which is a solution of $X^{2}-a X-b$ with $\operatorname{deg} a=d>0$ and $\operatorname{deg} b=0$. Then $j^{\mathrm{qt}}(f)$ has precisely $d=\operatorname{deg} a=\log _{q}|\sqrt{D}|$ values, and they are

$$
j^{\mathrm{at}}(f)=\left\{j(f)_{l}:=\frac{1}{\frac{1}{T^{q}-T}-J(f)_{l}}\right\}_{l=0}^{d-1} .
$$

Proof. The conjugate solution satisfies $f^{\prime}=-b f^{-1}$ with $b \in \mathbb{F}_{q}^{\times}$, so $f^{\prime}$ is $\mathrm{GL}_{2}(A)$ equivalent to $f$, hence by modularity, $j^{\text {qt }}\left(f^{\prime}\right)=j^{\text {qt }}(f)$. Thus, in what follows, we will suppose $f$ satisfies $|f|>1$. As above, $\varepsilon=q^{-N d-l}$. We begin by noting that

$$
\sum_{\lambda \in \Lambda_{\varepsilon}^{\text {bas }}(f)} \lambda^{-n}=\zeta_{T, l}(n) \overline{\mathbf{Q}}_{N}^{-n}+\zeta_{T}(n) \sum_{i=1}^{\infty} \overline{\mathbf{Q}}_{N+i}^{-n} .
$$

Then if we define

$$
H_{N, l}(n):=\zeta_{T, l}(n) \overline{\mathbf{Q}}_{N}^{-n}+\sum_{\substack{\text { Conditions I,II,III } \\ c_{0}(T) \neq 0}}\left(\sum_{i=0}^{m} c_{i}(T) \overline{\mathbf{Q}}_{N+i}\right)^{-n}
$$


and

$$
H_{N}(n):=\zeta_{T}(n) \sum_{i=1}^{\infty} \overline{\mathbf{Q}}_{N+i}^{-n}+\sum_{\substack{\text { Conditions } \\ \text { I,II }}}\left(\sum_{i=1}^{m} c_{i}(T) \overline{\mathbf{Q}}_{N+i}\right)^{-n},
$$

we may re-write (8) as

$$
\tilde{J}_{\varepsilon}(f)=\frac{H_{N, l}\left(q^{2}-1\right)+H_{N}\left(q^{2}-1\right)}{\left(H_{N, l}(q-1)+H_{N}(q-1)\right)^{q+1}} .
$$

Replace in (9) each $\overline{\mathrm{Q}}_{N+i}$ by $\left(\bar{f}^{N+i+1}-\bar{f}^{\prime N+i+1}\right) / c \sqrt{D}$ using (4)): equivalently (since the numerator and denominator of (9) have homogeneous degree $1-q^{2}$ ) we may omit the constant $1 / c \sqrt{D}$ and simply replace $\overline{\mathrm{Q}}_{N+i}$ by $\bar{f}^{N+i+1}-\bar{f}^{\prime N+i+1}$. Then dividing out the numerator and denominator by $\bar{f}^{\left(1-q^{2}\right)(N+1)}$ yields

$$
\tilde{J}_{\mathcal{E}}(f)=\frac{\hat{H}_{N, l}\left(q^{2}-1\right)+\hat{H}_{N}\left(q^{2}-1\right)}{\left(\hat{H}_{N, l}(q-1)+\hat{H}_{N}(q-1)\right)^{q+1}},
$$

where

$$
\begin{aligned}
\hat{H}_{N, l}(n):= & \zeta_{T, l}(n)\left(1-\left(\bar{f}^{\prime} / \bar{f}\right)^{N+1}\right)^{-n} \\
& +\sum_{\substack{\text { Conditions I,II,III } \\
c_{0}(T) \neq 0}}\left(\sum_{i=0}^{m} c_{i}(T) \bar{f}^{i}\left(1-\left(\bar{f}^{\prime} / \bar{f}\right)^{N+i+1}\right)\right)^{-n}
\end{aligned}
$$

and

$$
\begin{aligned}
\hat{H}_{N}(n):= & \left\{\zeta_{T}(n) \sum_{i=1}^{\infty} \bar{f}^{-n i}\left(1-\left(\bar{f}^{\prime} / \bar{f}\right)^{N+i+1}\right)^{-n}\right. \\
& +\sum_{\substack{\text { Conditions } \\
\text { I,II }}}\left(\sum_{i=1}^{m} c_{i}(T) \bar{f}^{i}\left(1-\left(\bar{f}^{\prime} / \bar{f}\right)^{N+i+1}\right)\right)^{-n} .
\end{aligned}
$$

Since $\left|\bar{f}^{\prime} / \bar{f}\right|<1$,

$$
1-\left(\bar{f}^{\prime} / \bar{f}\right)^{N+i+1} \longrightarrow 1
$$

as $N \rightarrow \infty$, uniformly in $i$, and it follows that

$$
\lim _{N \rightarrow \infty} \hat{H}_{N, l}(n)=H_{l}(n), \quad \lim _{N \rightarrow \infty} \hat{H}_{N}(n)=H(n)
$$

and therefore

$$
\lim _{N \rightarrow \infty} \tilde{J}_{q^{-N d-l}}(f)=\tilde{J}(f)_{l}
$$

Note 1. In the number field case, PARI GP experiments 3 performed on fundamental quadratic units $\theta$ indicate that $j^{\mathrm{qt}}(\theta)$ appears to have $D$ values, where $D$ is the corresponding fundamental discriminant. 


\section{Arbitrary Real Quadratics}

Let $h \in k_{\infty}-k$ be an arbitrary quadratic. By the Dirichlet Unit Theorem [1], the group of units in the quadratic extension $k(h)$ is isomorphic to $\mathbb{F}_{q}^{\times} \times \mathbb{Z}$. Thus there exists a unit $f$ such that $k(h)=k(f)$, i.e., $h$ can be written in the form

$$
h=\frac{x+y f}{z}, \quad x, y, z \in A,
$$

where $f$ satisfies $X^{2}-a X-b=0, d=\operatorname{deg}(a)>0$ and $\operatorname{deg}(b)=0$.

Note that for $h$ and $f$ as in (11) and $\varepsilon$ satisfying $|z| \varepsilon<1$, we have the following inclusions of $\mathbb{F}_{q}$-vector spaces

$$
z \Lambda_{|y|^{-1} \varepsilon}(f) \subset \Lambda_{\varepsilon}(h) \subset y^{-1} \Lambda_{|z| \varepsilon}(f) .
$$

The first inclusion follows upon noting that if $z \lambda \in z \Lambda_{|y|^{-1} \varepsilon}(f)$ we have

$$
\|(z \lambda) h\|=\left|(z \lambda) h-\left(y \lambda^{\perp}+\lambda x\right)\right|=\left|\lambda(x+y f)-\left(y \lambda^{\perp}+\lambda x\right)\right|=|y|\left|\lambda f-\lambda^{\perp}\right|<\varepsilon .
$$

The second inclusion follows from noting that if $\lambda \in \Lambda_{\varepsilon}(h), y \lambda \in \Lambda_{|z| \varepsilon}(f)$, as

$$
\|(y \lambda) f\|=\left|(y \lambda) f-\left(\lambda^{\perp} z-\lambda x\right)\right|=\left|\lambda(z h-x)-\left(\lambda^{\perp} z-\lambda x\right)\right|=|z|\left|\lambda h-\lambda^{\perp}\right|<|z| \varepsilon .
$$

Lemma 2. The inclusion of $\mathbb{F}_{q}$-vector spaces

$$
z \Lambda_{|y|^{-1} \varepsilon}(f) \subset \Lambda_{\varepsilon}(h)
$$

has index bounded by a constant which depends only on $y$ and $z$.

Proof. By (12), it suffices to show that the induced inclusion

$$
z \Lambda_{|y|^{-1} \mathcal{\varepsilon}}(f) \subset y^{-1} \Lambda_{|z| \varepsilon}(f)
$$

is of index bounded by a constant which only depends on $y, z$. For any $\delta<1$, denote

$$
\Lambda_{\delta}^{2}(f):=\left\{\left(\lambda, \lambda^{\perp}\right) \in A^{2} \mid \lambda \in \Lambda_{\delta}(f)\right\} .
$$

Note that the map $\lambda \mapsto\left(\lambda, \lambda^{\perp}\right)$ induces an isomorphism $\Lambda_{\delta}(f) \cong \Lambda_{\delta}^{2}(f)$ of $\mathbb{F}_{q^{\text {-vector }}}$ spaces: this in fact follows from Proposition 1 of [2]. In turn, we obtain an induced isomorphism of $y^{-1}$-rescalings

$$
y^{-1} \wedge_{\delta}(f) \cong y^{-1} \wedge_{\delta}^{2}(f)
$$

Taking $\delta=|z| \mathcal{E}$, the corresponding isomorphism takes $z \Lambda_{|y|^{-1} \mathcal{\varepsilon}}(f)$ to $z \Lambda_{|y|^{-1} \mathcal{\varepsilon}}^{2}(f)$. Thus

$$
y^{-1} \Lambda_{|z| \varepsilon}(f) / z \Lambda_{|y|^{-1} \varepsilon}(f) \cong y^{-1} \Lambda_{|z| \varepsilon}^{2}(f) / z \Lambda_{|y|^{-1} \mathcal{\varepsilon}}^{2}(f) .
$$

On the other hand, the natural map

$$
y^{-1} \Lambda_{|z| \varepsilon}^{2}(f) / z \Lambda_{|y|^{-1} \mathcal{\varepsilon}}^{2}(f) \hookrightarrow\left(y^{-1} A\right)^{2} /(z A)^{2}
$$

is injective: for if $\left(\lambda_{1}, \lambda_{1}^{\perp}\right),\left(\lambda_{2}, \lambda_{2}^{\perp}\right) \in \Lambda_{|z| \varepsilon}^{2}(f)$ satisfy

$$
y^{-1}\left(\lambda_{1}, \lambda_{1}^{\perp}\right)-y^{-1}\left(\lambda_{2}, \lambda_{2}^{\perp}\right)=z\left(\beta_{1}, \beta_{2}\right) \in(z A)^{2},
$$

then since $z\left(\beta_{1}, \beta_{2}\right) \in y^{-1} \Lambda_{|z| \varepsilon}^{2}(f)$ (being a difference of elements of the latter),

$$
\left|\left(y z \beta_{1}\right) f-y z \beta_{2}\right|<|z| \varepsilon
$$

which implies $\left(\beta_{1}, \beta_{2}\right) \in \Lambda_{|y|^{-1} \varepsilon}^{2}(f)$. Therefore the index of $z \Lambda_{|y|^{-1} \varepsilon}(f)$ in $y^{-1} \Lambda_{|z| \varepsilon}(f)$ is bounded by $\operatorname{dim}_{\mathbb{F}_{q}}\left(\left(y^{-1} A\right)^{2} /(z A)^{2}\right)$. This proves the Lemma.

Theorem 2. Let $h \in k_{\infty}-k$ be quadratic. Then $\# j^{\mathrm{qt}}(h)<\infty$. 
Proof. By Lemma 2, for each $\varepsilon$, we may find $\lambda_{1, \varepsilon}=0, \ldots, \lambda_{r, \varepsilon} \in \Lambda_{\varepsilon}(h), r<M=$ $M(y, z)$, such that

$$
\Lambda_{\varepsilon}(h)=\left[z \Lambda_{|y|^{-1} \varepsilon}(f)+\lambda_{1, \varepsilon}\right] \bigsqcup \cdots \bigsqcup\left[z \Lambda_{|y|^{-1} \varepsilon}(f)+\lambda_{r, \varepsilon}\right] .
$$

Thus, we have

$$
\begin{aligned}
\tilde{J}_{\mathcal{E}}(h)=\frac{\zeta_{h, \varepsilon}\left(q^{2}-1\right)}{\zeta_{h, \varepsilon}(q-1)^{q+1}} & =-\frac{\sum_{\lambda \in \Lambda_{\varepsilon}(h)-0} \lambda^{1-q^{2}}}{\left(\sum_{\lambda \in \Lambda_{\varepsilon}(h)-0} \lambda^{1-q}\right)^{q+1}} \\
& =-\frac{\sum_{i=1}^{r} \sum_{\lambda \in \Lambda_{|y|-1_{\varepsilon}}(f)}\left(\lambda+\lambda_{i, \varepsilon} / z\right)^{1-q^{2}}}{\left(\sum_{i=1}^{r} \sum_{\lambda \in \Lambda_{|y|-1}(f)}\left(\lambda+\lambda_{i, \varepsilon} / z\right)^{1-q}\right)^{1+q}} .
\end{aligned}
$$

By (12), for $i \neq 1$,

$$
\lambda_{i, \varepsilon} \in y^{-1} \Lambda_{|z| \varepsilon}(f)-z \Lambda_{|y|^{-1} \mathcal{\varepsilon}}(f),
$$

so we may write

$$
\lambda_{i, \varepsilon}=y^{-1} \sum_{j=m}^{m^{\prime}} c_{i j, \varepsilon}(T) \overline{\mathbf{Q}}_{i},
$$

where $\operatorname{deg}\left(c_{i j}(T)\right) \leq d-1$ and $m$ is the smallest index so that $\overline{\mathbf{Q}}_{m} \in \Lambda_{|z| \varepsilon}(f)$. Note that by (14) and Lemma 2, the difference $m^{\prime}-m$ has a uniform bound which is independent of $\varepsilon$. In particular, the set of all possible coefficients $c_{i j, \varepsilon}(T)$ is finite in number.

Consider a sequence $\left\{q^{-N d-l}\right\}_{N>1}$ of values for $\varepsilon$ giving a limiting value for $j^{\text {qt }}(f)$ as described in Theorem 1: recall that the limit is calculated by using Binet's formula to replace occurrences of $\overline{\mathrm{Q}}_{N+i}$ by $\bar{f}^{N+i+1}$ in $\tilde{J}_{\mathcal{\varepsilon}}(f)$ and dividing out by $\bar{f}^{\left(1-q^{2}\right)(N+1)}$. We apply exactly the same process to $\tilde{J}_{\mathcal{E}}(h)$ : and in view of (15) we obtain approximations

$$
z^{-1} \frac{\lambda_{i, \varepsilon}}{\bar{f}^{N+1}} \sim(y z)^{-1} \sum_{j=m}^{m^{\prime}} c_{i j, \varepsilon}(T) \bar{f}^{j-N}, \quad m, m^{\prime} \geq 0
$$

where $\operatorname{deg} c_{i j, \varepsilon}(T) \leq d-1$. Therefore, the set of possible limits of the $z^{-1} \frac{\lambda_{i, \varepsilon}}{f^{N+1}}$ as $N \rightarrow \infty$ is contained in the finite set

$$
\left\{(y z)^{-1} \sum_{j=-n}^{n^{\prime}} c_{j}(T) \bar{f}^{j} \mid \operatorname{deg}\left(c_{j}(T)\right) \leq d-1\right\}
$$

where $n^{\prime}+n=m^{\prime}-m$. Thus, within the family $\left\{\varepsilon=q^{-N d-l}\right\}_{N>1}$, there are only finitely many possible limits of sub-sequences of $\left\{\tilde{J}_{\mathcal{E}}(h)\right\}$ giving rise to elements of $j^{\mathrm{qt}}(h)$. This proves the Theorem.

\section{REFERENCES}

[1] Cohn, P.M., Algebraic Numbers and Algebraic Functions. Chapman and Hall/CRC, London, 1991.

[2] Demangos, L \& Gendron, T.M., Quantum $j$-Invariant in positive characteristic I: definitions and convergence.

[3] Castaño Bernard, C. \& Gendron, T.M., Modular invariant of quantum tori. Proc. Lond. Math. Soc. 109 (2014), Issue 4, 1014-1049. 
Instituto de Matemáticas - Unidad Cuernavaca, Universidad Nacional Autonoma de México, Av. Universidad S/N, C.P. 62210 Cuernavaca, Morelos, México

Current address: Department of Mathematical Sciences (Mathematics Division), University of Stellenbosch, Private Bag X1, Matieland 7602, South Africa

E-mail address: 1.demangos@gmail.com

Instituto de Matemáticas - Unidad Cuernavaca, Universidad Nacional Autonoma de México, Av. Universidad S/N, C.P. 62210 Cuernavaca, Morelos, México

E-mail address: tim@matcuer.unam.mx 